\title{
Three-Dimensional Pattern of Enkephalin-like Immunoreactivity in the Caudate Nucleus of the Cat
}

\author{
Philip M. Groves, Maryann Martone, ${ }^{1}$ Stephen J. Young, and David M. Armstrong' \\ Departments of Psychiatry and of 'Neurosciences, UCSD Medical Center, San Diego, California 92093
}

Recent anatomical investigations of the mammalian neostriatum have uncovered discrete neurochemical zones characterized by low levels of AChE and high levels of enkephalin-like immunoreactivity (ELI) compared with the surrounding neostriatal tissue. These regions, termed striosomes, which appear as patches in individual sections, have been associated with specific afferent and efferent pathways that differ from those of the surrounding neostriatal tissue.

In the present study, the 3-dimensional distribution of these enkephalin-rich compartments in the caudate nucleus of the adult cat was investigated using computer-assisted 3-dimensional reconstruction techniques. Series consisting of coronal, sagittal, and horizontal tissue sections were obtained. In individual sections, discrete patches of intense enkephalin-like immunoreactivity appeared against a lighter-staining background.

Three-dimensional reconstructions revealed that these patches overlapped across sections to form a highly interconnected structure within the head and body of the caudate nucleus. Several structural features were evident in these reconstructions that appeared to be similar across animals. One consistent pattern was the formation of enkephalinlabeled finger-like structures radiating from the ventricular edge diagonally across the width of the nucleus. Smaller crossbridges were seen connecting the fingers, which often resulted in the formation of claws or rings of enkephalin-like immunoreactivity. These elements appear to align across planes to form a lattice-like structure outlining continuous regions of matrix. This structure may provide a basis for the orderly interaction between the patch and matrix compartments.

The mammalian neostriatum, consisting of the caudate nucleus and putamen, is known to be critical for the execution of voluntary movement and may be partly responsible for the "automatic execution of learned motor plans" (Marsden, 1982). Unlike the cerebral and cerebellar cortices, which exhibit clear organizational anatomical features in the light microscope, such as a characteristic layered appearance, the neostriatum appears

\footnotetext{
Received Apr. 16, 1987; revised July 29, 1987; accepted July 29, 1987.

This work was supported in part by contract N000-14-85-K-0699 from the Office of Naval Research, Grant AG 05344 (to D.M.A.) from the National Institute of Aging, and Grant DA 02854 and Research Scientist Award DA 00079 (to P.M.G.) from the National Institute on Drug Abuse. We would like to thank Drs. Lawrence Ryan and James Tepper for valuable discussions.

Correspondence should be addressed to P. M. Groves, Ph.D., Department of Psychiatry (M-003), UCSD School of Medicine, San Diego, CA 92093.

Copyright (C) 1988 Society for Neuroscience $0270-6474 / 88 / 030892-09 \$ 02.00 / 0$
}

as a relatively homogeneously distributed cell mass. The majority of neostriatal neurons belong to a single morphological class, the common spiny neuron (Kemp and Powell, 1971). The absence of any obvious structural differentiation and the predominance of a single morphological cell type has made it difficult to determine what organizational principles might underlie information processing in the neostriatum.

Despite the relatively homogeneous appearance of neostriatal tissue in Nissl preparations, it became apparent that considerable biochemical heterogeneity existed within the neostriatum, particularly in developing animals (Olson et al., 1972; Butcher and Hodge, 1976). An important advance in understanding the neostriatum was the discovery that discrete neurochemical compartments can be identified in sections of neostriatum of both adult and developing animals stained for AChE (Graybiel and Ragsdale, 1978). In their work, Graybiel and Ragsdale observed discrete zones, $0.2-0.6 \mathrm{~mm}$ wide, that exhibited low AChE activity relative to the surrounding neostriatal tissue. These zones of low AChE activity, termed striosomes or patches, were later found to correspond to similar mosaic distributions described in the striatum for other neurotransmitter markers, neuropeptides, receptors, patterns of afferent termination, and histogenesis during development (Donoghue and Herkenham, 1986; Graybiel et al., 1981, 1986; Ragsdale and Graybiel, 1981, 1984; Goedert et al., 1983; Gerfen, 1984; Graybiel, 1984a; Nastuk and Graybiel, 1985; O'Brien et al., 1986; Sandell et al., 1986). For example, when alternate sections processed for $\mathrm{AChE}$ and enkephalin were compared, the AChE-poor zones appeared in exact spatial register with patches of high enkephalin-like immunoreactivity (Graybiel et al., 1981). Similarly, the dopamine enriched islands described in developing animals appear in precisely the same regions of neostriatum later occupied by the AChE-poor striosomes (Graybiel, 1984a). Other striatal afferent as well as efferent connections appear to be differentiated with respect to these compartments (Graybiel et al., 1979; Herkenham and Pert, 1981; Ragsdale and Graybiel, 1981; Gerfen, 1984, 1985; Donoghue and Herkenham, 1986; Malach and Graybiel, 1986).

While these patch compartments have been observed to overlap in adjacent sections throughout the neostriatum, suggesting that thcy form parts of larger structures (Graybiel et al., 1981; Graybiel, 1983), the 3-dimensional organization of this system has not been assessed. Recent applications of computer programs for 3-dimensional reconstruction of serial tissue sections has made it possible to visualize highly complex structures such as synaptic endings and their postsynaptic targets (Groves, 1980; Groves and Wilson, 1980; Groves and Linder, 1983; Wilson et al., 1983). In the present study, these computer techniques were employed to investigate the 3-dimensional organization of 
patches of enkephalin-like immunoreactivity seen in individual sections of striatal tissue. We have found that these patches are not isolated regions within the neostriatal parenchyma, but rather are distributed in a remarkably organized, often interconnected 3-dimensional lattice with anatomical features that appear to be consistent from brain to brain. This lattice may represent an important structural framework for understanding the functional organization of the mammalian neostriatum. A preliminary report of this work has appeared in abstract form (Martone et al., 1986).

\section{Materials and Methods}

Immunocytochemistry. Adult mongrel cats were anesthetized deeply with pentobarbitol (Nembutol) and perfused transcardially with $500 \mathrm{ml}$ $0.1 \mathrm{M}$ sodium phosphate buffer (pH 7.2), followed by $1-2$ liters of $4 \%$ paraformaldehyde or $4 \%$ paraformaldehyde and $0.1 \%$ glutaraldehyde in $0.1 \mathrm{~m}$ phosphate buffer. Blocks of tissue containing the entire rostrocaudal extent of the neostriatum were postfixed for $24-48 \mathrm{hr}$ at $4^{\circ} \mathrm{C}$ in the same fixative with no glutaraldehyde and then transferred to $20 \%$ buffered sucrose for an additional $48 \mathrm{hr}$ or until the tissue sank. Tissue blocks were frozen and $50-\mu \mathrm{m}$-thick serial sections were cut on a sliding microtome in the coronal, horizontal, or sagittal plane and collected in $0.1 \mathrm{~m}$ phosphate buffer. Those sections not processed immediately for immunocytochemistry were transferred to a solution of glycerol-ethylene glycol in $0.1 \mathrm{~m}$ phosphate buffer and stored at $-20^{\circ} \mathrm{C}$.

Series containing every section, every other, or every third section were processed for immunocytochemistry with antibodies against leuenkephalin using a modification (Armstrong et al., 1981) of Sternberger's (1979) peroxidase-antiperoxidase (PAP) technique. The following steps were included in this procedure: (1) $30 \mathrm{~min}$ incubation in $3 \%$ normal goat serum (NCS) in $0.1 \mathrm{~m}$ Tris-HCl buffer containing $0.9 \%$ saline (TBS, pH 7.4) and $0.25 \%$ Triton $\mathrm{X}-100$; (2) $48 \mathrm{hr}$ incubation at $4^{\circ} \mathrm{C}$ with antiserum raised in rabbit against leu-enkephalin (Immunonuclear) diluted 1:2000 with TBS-Triton containing 1\% NGS; (3) $60 \mathrm{~min}$ incubation with goat anti-rabbit IgG (Cappel) diluted 1:300 with TBS-1\% NGS; (4) 60 min incubation with rabbit PAP complex (Cappel) diluted 1:400 with TBS-1\% NGS; (5) 10 min incubation in 3-3'-diaminobenzidine $(40 \mathrm{mg} / 150 \mathrm{ml})$ in $0.1 \mathrm{M}$ Tris- $\mathrm{HCl}(\mathrm{pH} 7.4)$ containing $0.01 \%$ hydrogen peroxide and $0.004 \%$ nickel chloride. Addition of the nickel chloride results in a purple reaction product and serves to intensify the labeling. Two $10 \mathrm{~min}$ washes were performed between each step. All incubations were performed at room temperature unless otherwise noted. Sections were mounted on gelatin-coated slides, dehydrated through a graded series of alcohols, cleared, and coverslipped.

Specificity of the immunocytochemical labeling procedure was assessed by omitting the primary antiserum from step 2 or substituting the primary antiserum with antiserum preabsorbed with $250 \mu \mathrm{g} / \mathrm{ml} \mathrm{of}$ synthetic leu-enkephalin (Penninsula). Both procedures resulted in a complete lack of specific staining within the caudate nucleus. However, since it is possible that the primary antiserum may cross-react with some pro-hormone or structurally similar molecule, the term enkephalin-like immunoreactivity (ELI) will be used throughout this report.

Three-dimensional reconstructions. As others have observed, ELI was distributed in the caudate nucleus of the cat in densely stained, irregularly shaped patches (Fig. 1). The intensity of immunolabeling within these patches was in sharp contrast to that in the surrounding matrix over much of the caudate nucleus. Because the patches were considerably less distinct in the putamen and at more posterior levels of the caudate nucleus, only those patches within the head and body of the caudate nucleus were reconstructed. The boundary of the caudate nucleus and the outlines of the patches of intense ELI were drawn for each section using a $1 \times$ objective on a Leitz Ortholux II microscope with camera lucida attachment. The ventral boundary of the caudate nucleus was defined according to the criterion of Berman and Jones (1982). Major blood vessels were also included in the drawings to aid in registration.

The outlines of the patches and the boundaries of the caudate nucleus along with fiducial marks for each section were digitized using a highresolution tracing tablet (Sumagraphics Supergrid) and an IBM PC-ATcompatible computer system. Fiducial marks were determined by overlapping consecutive drawings on a light box and aligning the sections using the contours of the caudate nucleus and several major blood vessels for registration. Data input and reconstruction programs were used to align the sections using a least-squares algorithm and to generate 3-dimensional color reconstructions at a specified angle of view with hidden-line suppression and depth cuing. Images could be rotated on the $x, y$, and $z$ axes. Black-and-white images were obtained by photographing the reconstructions directly off the computer screen. The data input and reconstruction programs used were modifications of those developed by Young et al. (1987).

Reconstructions were obtained from both caudate nuclei from 5 brains: 2 from series of coronal sections, 2 from horizontal series, and 1 from sagittal series. Coronal and horizontal reconstructions were based on every other or every third section, while the sagittal reconstruction contained every section.

\section{Results}

In individual sections, peroxidase reaction product was localized within numerous varicose proccsscs distributcd in densely stained patches, $200-500 \mu \mathrm{m}$ in diameter (Fig. 1). No perikarya containing ELI were seen. Reconstructions obtained from coronal, horizontal, and sagittal section series revealed that the patches of ELI seen in individual sections overlap across sections to form a highly interconnected lattice-like structure throughout the head and body of the caudate nucleus. In several instances, a series of overlapping patches could be followed through 50 or more sections (approximately $5 \mathrm{~mm}$ ). A considerable degree of interconnection existed between patches, though it did not appear that they formed a single large network. Examination of these reconstructions revealed that, although the form of these networks is complex, certain regularly repeating structural features are apparent.

Reconstructions based on coronal and horizontal sections are shown in Figures 2-5. A consistent feature of these reconstructions is the presence of regularly spaced "fingers" of ELI, approximately $300 \mu \mathrm{m}$ in width, radiating from the ventricular edge ventrolaterally across the width of the caudate nucleus to the medial edge of the internal capsule. Six fingers are clearly visible in the reconstruction based on coronal sections (every third section) shown in Figure 2 (see arrows) and at a different angle of view in Figure 3. The number of fingers does, however, vary at different anterior-posterior levels. These fingers are also visible in reconstructions based on horizontal sections (Figs. 4, 5 , see cover illustration) and sagittal sections (not shown). Fingers were generally separated by distances of $400-700 \mu \mathrm{m}$ in both the coronal and horizontal planes, although the separation was not always constant along the length of adjacent fingers. These fingers were most clearly defined at more anterior levels of the striatum and were generally larger in diameter anteriorly $(350 \mu \mathrm{m})$ than posteriorly $(250 \mu \mathrm{m})$. The radial pattern of the fingers can be seen clearly in reconstructions based on a smaller number of horizontal sections (Fig. 5). At more anterior levels, the fingers generally curved from front to back, while at more posterior levels, they appeared to curve rostrally, almost perpendicular to the coronal plane. This change in orientation is consistent with the observation that individual patches seen in coronal sections change from large, diagonally oriented structures at anterior levels to smaller, circular profiles at posterior levels (Graybiel et al., 1981).

Numerous ELI crossbridges, which were generally smaller in width than the main fingers, were observed linking these fingers together in the coronal, horizontal, and sagittal planes (see large arrow, Fig. 5). Often this resulted in the formation of rings or "claws" of ELI surrounding areas of matrix devoid of patches. As seen in the stereo pairs in Figures 3 and 4, which show reconstructions based on coronal and horizontal sections, re- 


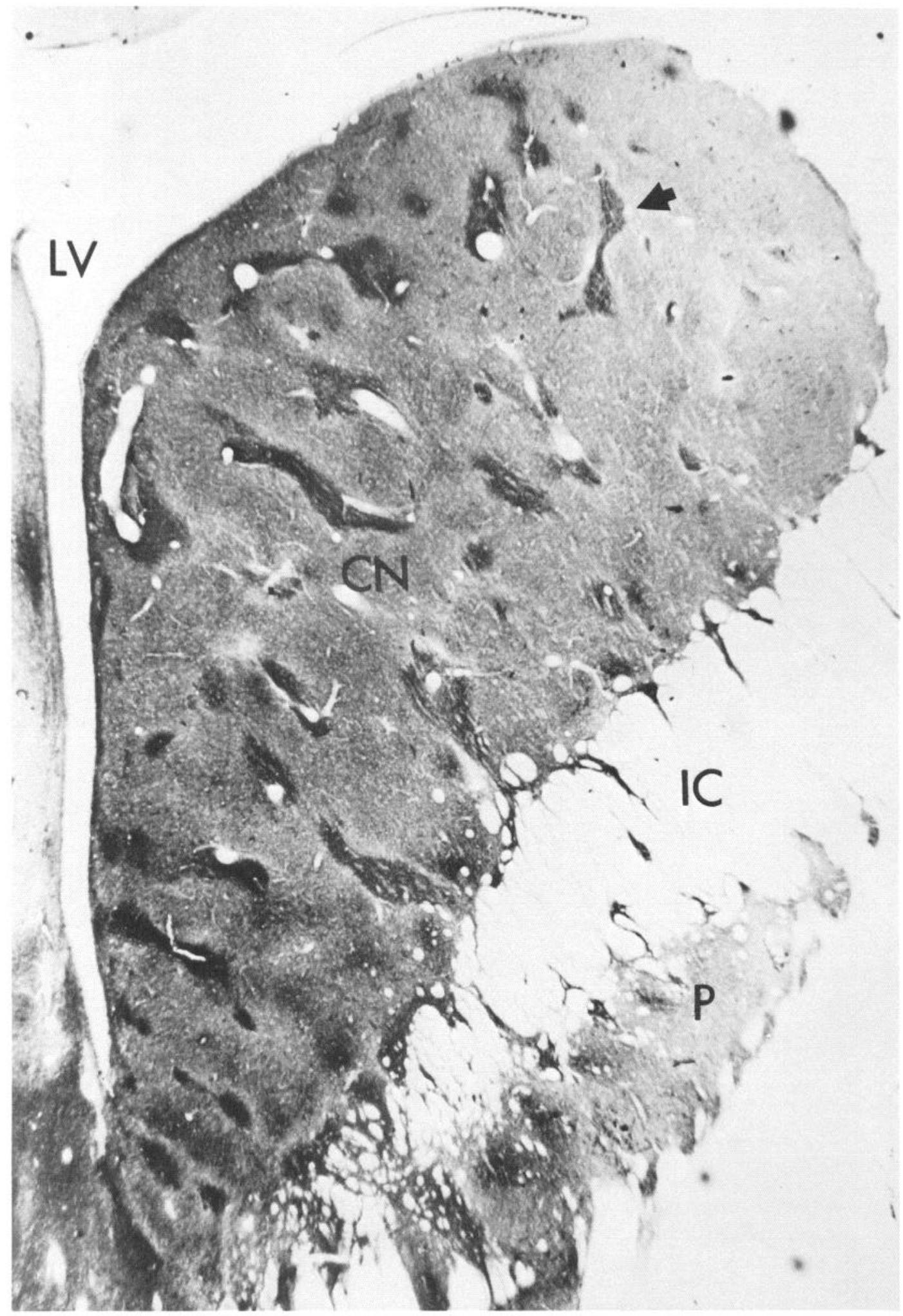

Figure 1. Low-power photomicrograph of ELI in cat striatum showing patches of intense ELI (arrow). CN, caudate nucleus; IC, internal capsule; $L V$, lateral ventricle; $P$, putamen.

spectively, the fingers of ELI connected by crossbridges appeared to be aligned in both the coronal and horizontal planes. Because of this regularity, continuous regions of matrix could be seen running between the fingers in the anterior-posterior, medial- lateral, and dorsal-ventral dimensions, giving the impression of a 3-dimensional lattice. This arrangement is represented schematically in Figure 6. Most striking, in coronal reconstructions, the rings and claws of ELI formed by the intersection of the 


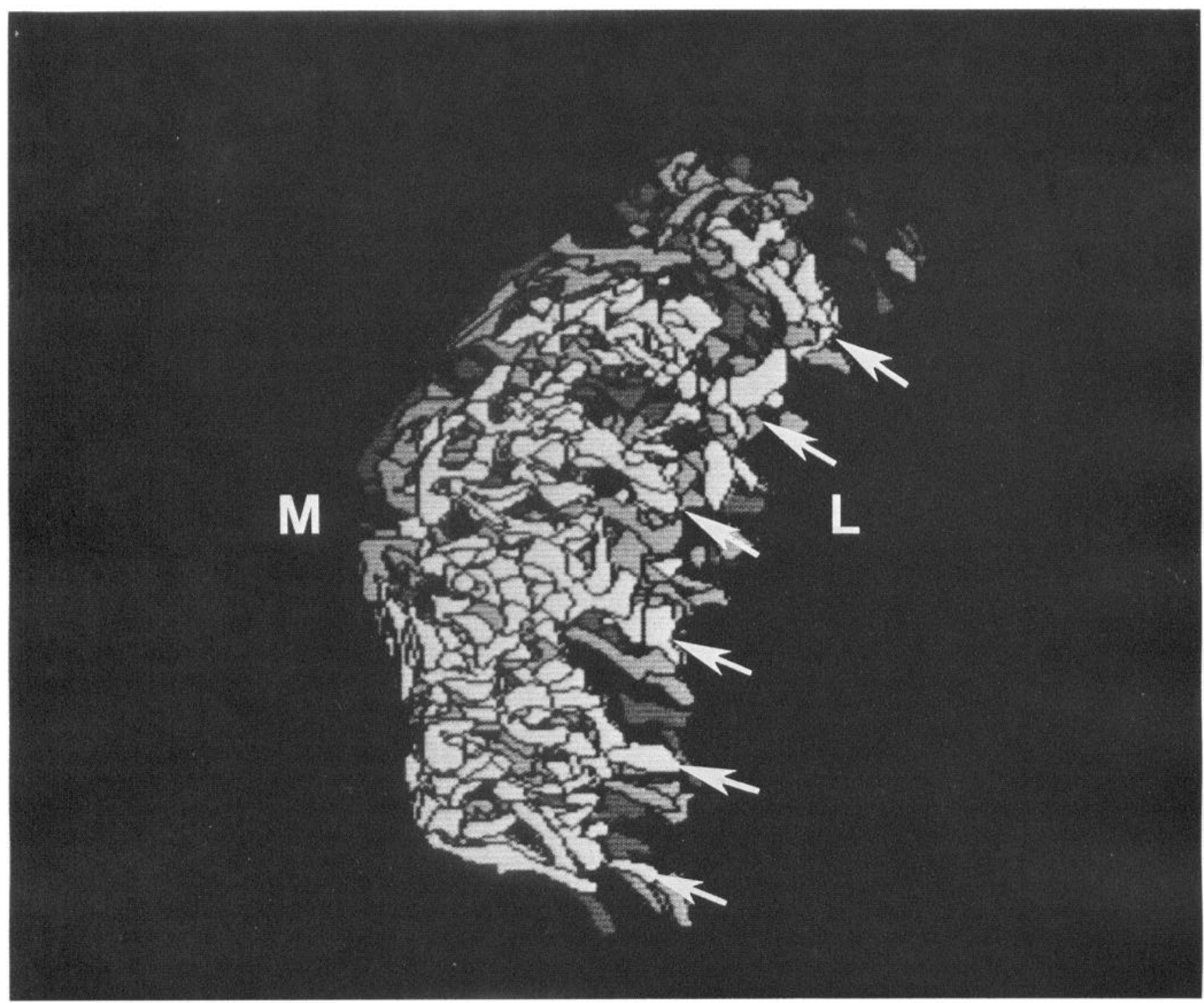

Figure 2. Reconstruction of enkephalin patches based on 58 coronal sections (every third section) through the head of the caudate nucleus of the left hemisphere viewed from the front. Arrows point to fingers of ELI seen radiating from the medial edge across the width of the caudate nucleus. In this and subsequent figures, sections are shaded from front to back so that more posterior structures appear darker. $M$, medial; $L$, lateral.

fingers and crossbridges appeared to line up across sections to define longitudinal cylindrical domains of matrix ensheathed by tubes of ELI (Fig. 3, arrow). These domains could be followed for variable distances through the head of the caudate nucleus and varied in diameter from 200 to $1000 \mu \mathrm{m}$.

The diagonally oriented fingers and longitudinal tubes were observed in all brains examined regardless of plane of section and the number of sections used in the reconstruction. When reconstructions based on limited numbers of sections at comparable levels of the caudate nucleus were examined, a striking similarity was apparent between different cats in the overall organization of the network. Figure 7 shows 2 coronal reconstructions of a series covering approximately $1 \mathrm{~mm}$ through the anterior head of the caudate nucleus from the left hemisphere of 2 cats. Both the placement of the diagonal fingers of ELI as well as the rings of ELI have direct counterparts in the 2 images. Such correspondences were not always this clear, however, which may reflect slight differences in plane of sectioning between animals as well as individual differences. Although not shown, similar general correspondences were observed in the form of the network in the caudate nucleus between the 2 hemispheres in the same brain.

\section{Discussion}

The present study provides the first detailed 3-dimensional view of ELI patches in the caudate nucleus of the cat. The most striking feature in these 3-dimensional reconstructions is the presence of large fingers of ELI radiating diagonally across the medial-lateral extent of the caudate nucleus. Numerous crossbridges are also seen linking these fingers together in all dimensions. The pattern formed by these large fingers and interconnecting crossbridges suggests that a highly organized, lattice-like network of ELI may exist within the caudate nucleus. Preliminary comparisons suggest that the form of this network is similar from brain to brain. Because the AChE-poor zones have been shown to correspond to patches of ELI with few exceptions (Graybiel et al., 1981), it is likely that the striosmal compartment also takes this form. In fact, other researchers have proposed 

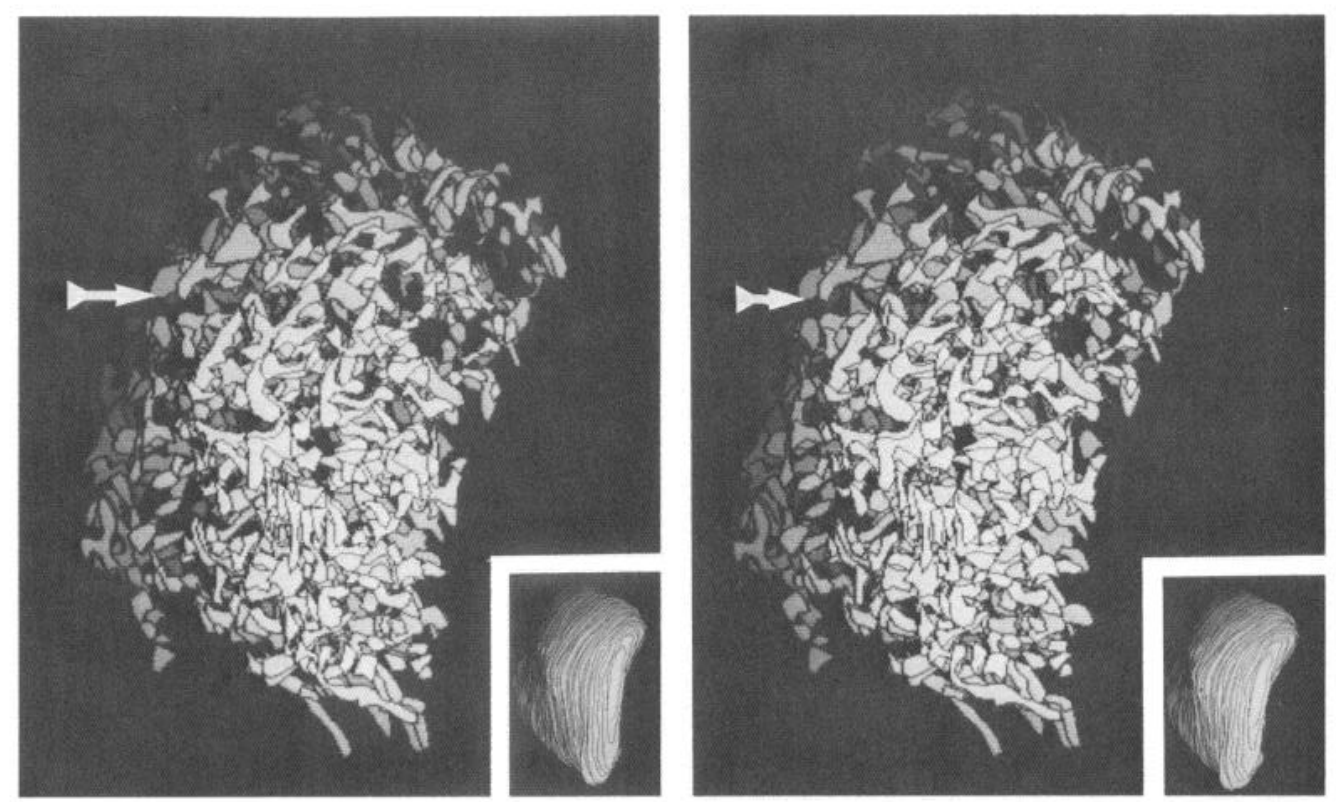

Figure 3. Stereo pair of reconstruction of enkephalin patches shown in Figure 2 at a different angle of view. The image is rotated to the right on the $y$ axis so that the medial or ventricular edge of the caudate nucleus is visible. Insert, Perimeter contours of the caudate nucleus from these same sections in the same rotation to aid in orientation. Arrow points to a cylindrical longitudinal domain of matrix defined by the network of ELI. The left figure is rotated $40^{\circ}$ to the right and the right figure is rotated $35^{\circ}$.

some of the features evident in our reconstructions, such as the diagonal fingers and ring-like elements, from an analysis of limited numbers of sections stained for a variety of striosomal markers(Goldman-Rakic, 1982; see discussion, Graybiel, 1984b; O'Brien et al., 1986).

Although the source of the ELI processes within the patches could not be identified in our material, other investigators have localized striatal enkephalin within a subpopulation of common spiny neurons (Pickel et al., 1980; Di Figlia et al., 1982; Penney et al., 1986). No extrinsic source of enkephalin has yet been identified in the striatum (Takagi, 1986). The spiny neuron is known to possess an extensive axon collateral field which is typically coextensive with its dendritic arborization (Wilson and
Groves, 1980). Since the dendritic fields of identified spiny neurons tend to be confined within patch boundaries (Penney et al., 1984; Bolam et al., 1985; Gerfen, 1985), it is likely that the ELI patches represent the dendritic or local axonal fields of these neurons. However, since enkephalinergic neurons are found both within patch and matrix compartments (Graybiel and Chesselet, 1984a), it remains to be determined what unique characteristics of patch regions result in the intense ELI staining observed within this compartment.

Several lines of evidence support the possibility that patch regions represent a specialized processing system within the striatum. Studies that have compared patterns of afferent termination with the striosomal compartment have shown that
Figure 4. Stereo pair of one reconstruction through the head of the caudate nucleus of the left hemisphere based on 80 horizontal sections (every other section) viewed from the back. The images are rotated to the left so that the lateral edge of the caudate nucleus is visible. The tail of the caudate can be seen at the top right of the figure. Insert, Perimeter contours of the caudate nucleus from these same sections in the same orientation. The images are rotated on the $y$ axis $60^{\circ}$ and $65^{\circ}$, respectively, to the left.
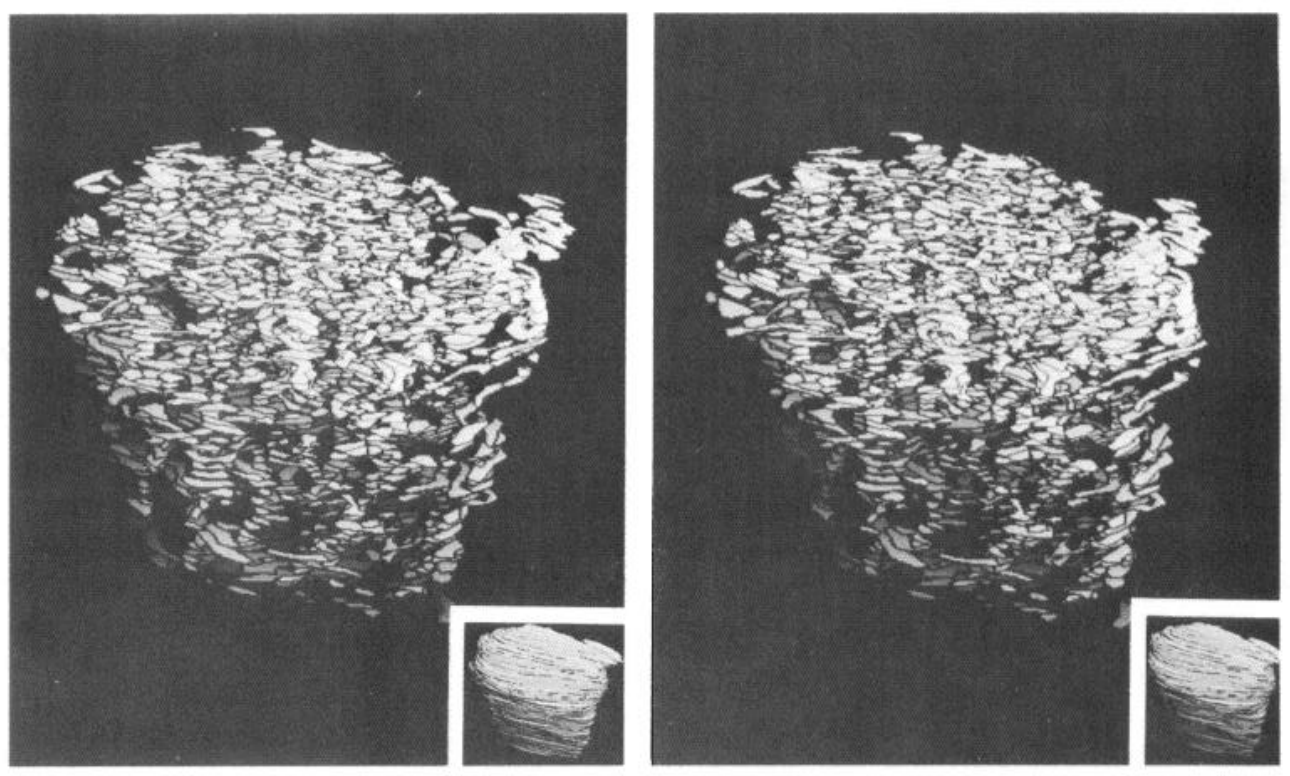

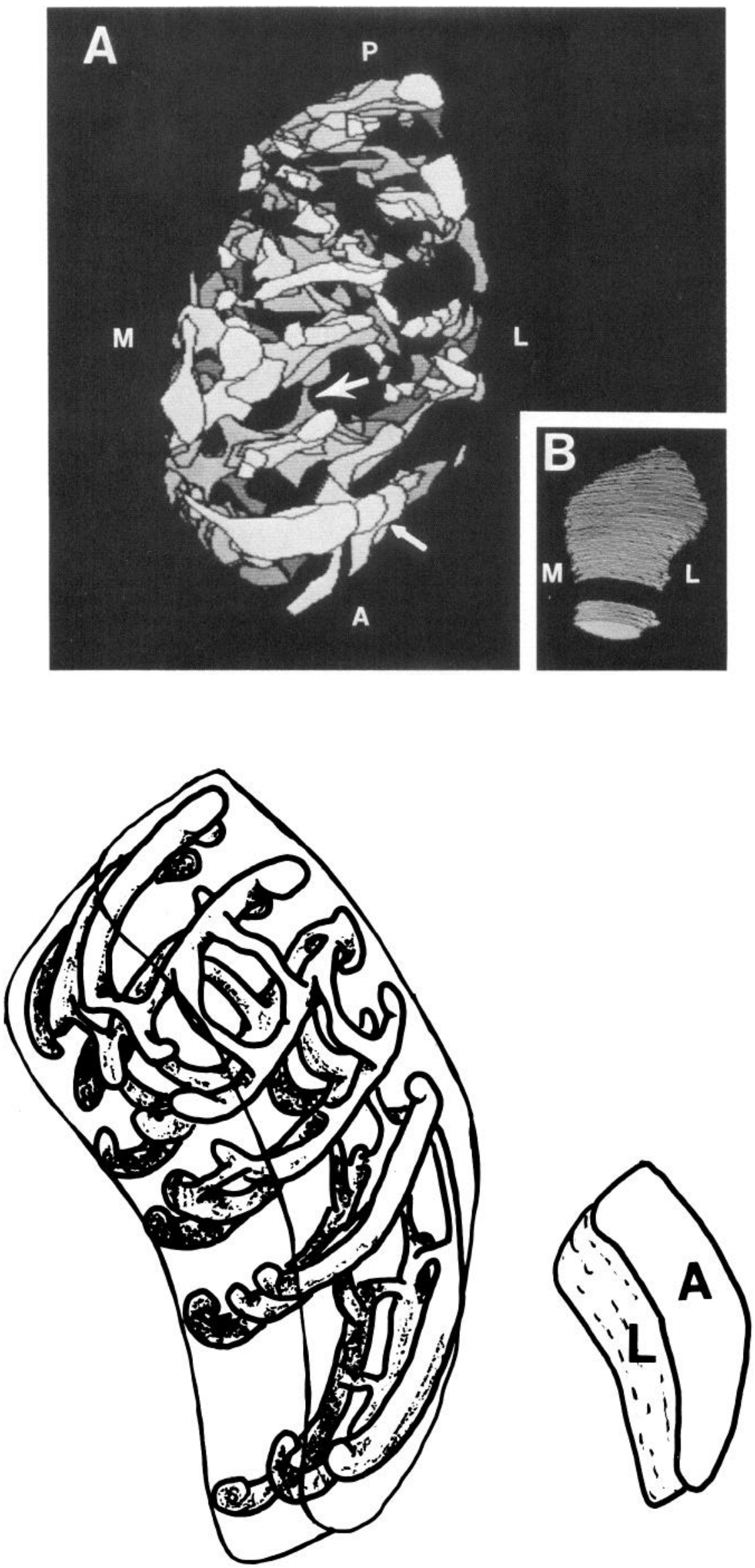

Figure 5. A, Partial reconstruction of enkephalin-like patches based on 10 horizontal sections viewed from above. Small arrow points to finger of ELI radiating from the medial edge; Large arrow points to smaller crossbridge that appears to be connecting 2 adjacent fingers. $B$, Reconstruction of entire series of caudate nucleus outlines rotated $90^{\circ}$ relative to $A$ to provide an edge-on view. Sections used in $A$ are shown in dark gray. $M$, medial; $L$, lateral; $A$, anterior; $P$, posterior.

Figure 6. Simplified schematic drawing of the structure of the network of ELI as it would appear in a region of the cat caudate nucleus several millimeters thick. Small outline gives the orientation of the figure. The illustration is based on computer-assisted 3-dimensional reconstructions derived from serial coronal, horizontal, and sagittal sections. $L$, lateral; $A$, anterior. 

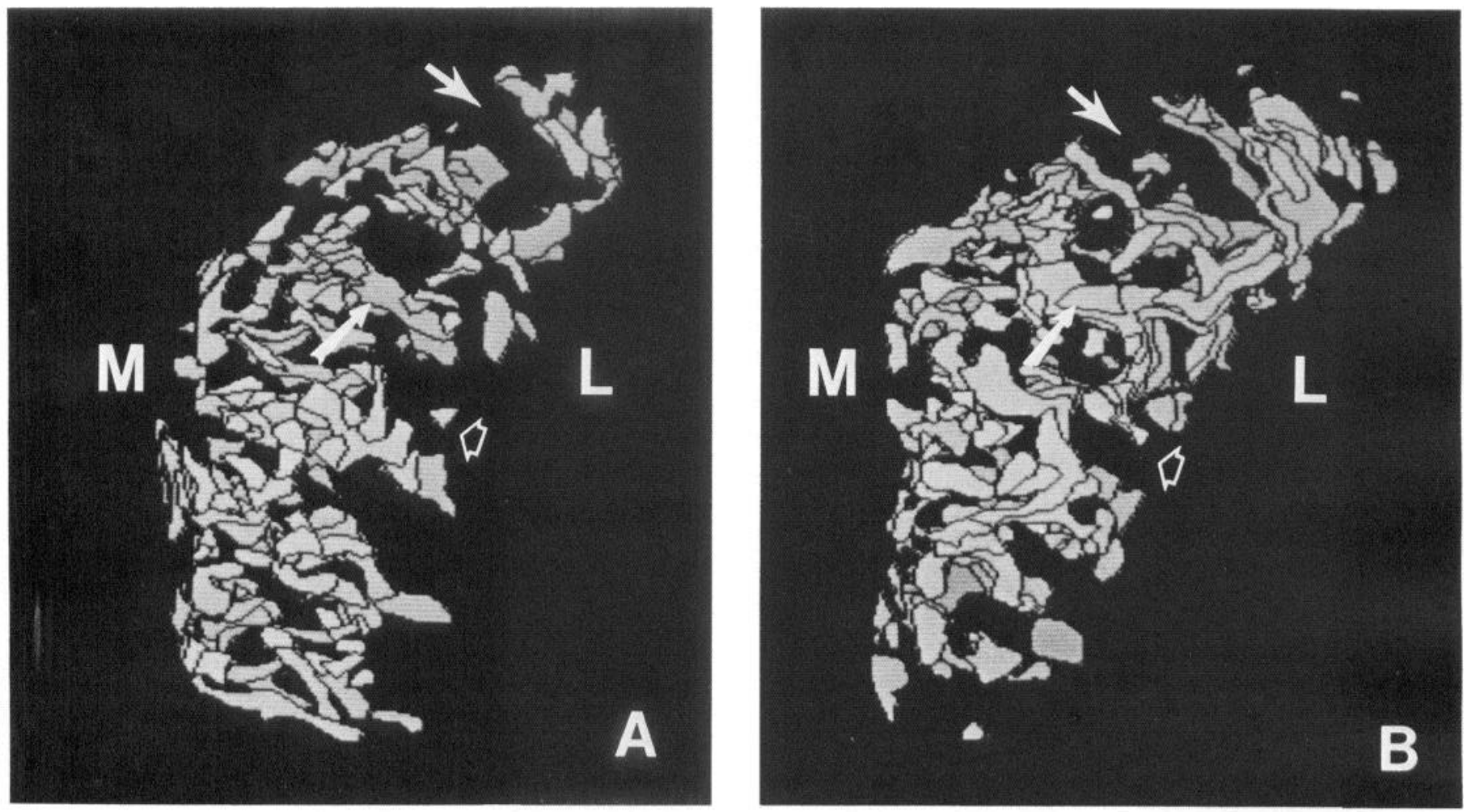

Figure 7. Reconstructions of patches of ELI from coronal sections from 2 different animals ( $A$, every third section; $B$, every other section). Both reconstructions cover approximately $1 \mathrm{~mm}$ through comparable levels of the head of the caudate nucleus. Arrows point to regions that appear to be similar in the 2 brains. $M$, medial; $L$, lateral.

projections from several cortical and thalamic areas terminate preferentially within either the striosomes or the extrastriosomal matrix (Goldman-Rakic, 1981; Herkenham and Pert, 1981; Ragsdale and Graybiel, 1981; Gerfen, 1984; Donoghue and Herkenham, 1986; Malach and Graybiel, 1986). Retrograde tracing studies suggest that the efferent pathways of patch and matrix may also be distinct. Neurons lying within the matrix project preferentially to globus pallidus and substantia nigra, pars reticulata (Graybiel et al., 1979; Gerfen, 1985), while neurons within the patches project to other regions, possibly the substantia nigra, pars compacta (Gerfen, 1985; Jimenez-Castellanos and Graybiel, 1985). Both intracellular injections of HRP (Penney et al., 1984) and retrograde tracing studies (Gerfen, 1985) have shown that the dendritic fields of labeled neurons lying within the patches are completely confined within the boundaries of the patch. Cell bodies containing substance $\mathrm{P}$ and dynorphin have also been found to be clustered in striosomes in the dorsal striatum (Graybiel and Chesselet, 1984b). In contrast to the view of the neostriatum as a homogeneously distributed cell mass (Kemp and Powell, 1971), the above data suggest that the network of ELI reflects an underlying regularity in the cytoarchitecture of the neostriatum. It remains to be determined how differences in the dimensions of the lattice, e.g., decreases in the diameter of the fingers posteriorly, relate to cellular features such as numbers of cells per finger and size of dendritic arbors.

The significance of such a lattice is not yet clear. The 3-dimensional structure of the striosomal compartment appears to be organized so as to interface in an orderly fashion with all areas of matrix. However, there is some question whether the striosomal system may be viewed as unitary. It appeared in our material and in others' (see discussion in Graybiel, 1984b) that the enkephalin patches were not interconnected in one large network but that 2 or more networks were likely present. In addition, it appears that both the afferent innervation and histochemistry of the striosomes may differ along the dorsal-ventral axis. For example, substance P-rich zones appear to characterize the striosomal compartment dorsally, while substance P-poor zones predominate ventrally (Graybiel et al., 1981; personal observations). In addition, certain prefrontal areas project preferentially to the striosomes dorsally and just miss them ventrally (Ragsdale and Graybiel, 1981, 1984). Further work is needed to determine whether separate striosomal networks exist, each with a unique functional, hodological, and histochemical identity, and/or whether individual networks gradually change in character along a particular dimension.

The observed distribution of ELI in diagonally and longitudinally oriented structures is consistent with several recent descriptions of the termination pattern of striatal afferents. The neostriatum receives afferent projections from all areas of the cerebral cortex, the intralaminar thalamic nuclei, and the substantia nigra (see Nauta and Domesick, 1984, for review). Early work on the cortical projection using classical silver degeneration techniques suggested that the neocortex projects in a simple topographic relationship to the underlying neostriatum, although considerable overlap occurs between adjacent projection zones (Kemp and Powell, 1970). However, recent studies using anterograde tracers indicate that several cortical areas project more extensively throughout the neostriatum than previously thought (Yetarian and Van Hoesen, 1978; Chapin et al., 1985; 
Selemon and Goldman-Rakic, 1985). Selemon and GoldmanRakic (1985), reporting on corticostriatal projections in the primate, found that all areas of association cortex examined projected to topographically restricted longitudinal strips thoughout the rostral-caudal extent of the caudate nucleus. When viewed in coronal sections, these termination patterns were seen as diagonal bands extending across the width of the caudate nucleus into the putamen. Projections from several thalamic nuclei also appear to terminate along widespread rostral-caudal areas of the striatum (Kalil, 1978; Beckstead, 1984). Interestingly, Kalil (1978) described "longitudinally oriented cylinders" of labeling in restricted sectors of the primate putamen after injections of tritiated amino acids into the centromedian thalamic nucleus.

Currently, with the exception of the dopaminergic input from the substantia nigra (Moon-Edley and Herkenham, 1984; Jimenez-Castellanos and Graybiel, 1985), the only other projection identified to the patch system has been from regions of prefrontal cortex (Ragsdale and Graybiel, 1981; Gerfen, 1984; Donoghue and Herkenham, 1986) and the amygdala (Ragsdale and Graybiel, 1984). All other cortical and thalamic regions examined so far have terminated within the matrix (Herkenham and Pert, 1981; Donoghue and Herkenham, 1986; Malach and Graybiel, 1986). The general longitudinal topography demonstrated for cortical and thalamic afferents to the striatum suggests that the inputs described above may be terminating within the longitudinal zones of matrix delineated by the ELI lattice. If this is the case, the lattice structure we described may reflect the subdivision of the neostriatal matrix into specialized processing modules and the means by which information processed within the patch system can be readily interfaced with them.

Although it is possible that the patch system may circumscribe large functional regions of matrix, recent evidence indicates that these regions are themselves further subdivided. A number of studies of corticostriatal afferents have shown that projections from several cortical areas terminate in a discontinuous patchy fashion within the matrix (Goldman and Nauta, 1977; Tanaka, et al., 1981; Selemon and Goldman-Rakic, 1985; Donoghue and Herkenham, 1986; Malach and Graybiel, 1986). Doublelabeling anterograde tracing studies have shown that even cortical regions that appear to terminate within a given striatal region interdigitate with, rather than overlap, one another (Selemon and Goldman-Rakic, 1985; Malach and Graybiel, 1986). For example, a recent study on the projection from primary somatosensory cortex to the caudate nucleus of the cat described a topographic patchy distribution of cortical efferents from superficial and deep cutaneous sensory modalities (Malach and Graybiel, 1986).

It will be important to determine the 3-dimensional organization of these "matrix patches" and their spatial relationship to the striosomal system. The 3-dimensional organization of the matrix patches comprising a given submodality may be similar to that of the striosomal system within a sector of the striatum. Additional understanding of the functioning of the striatum and its pathways should arise, for example, by examining whether the striosomal system interfaces with all submodalities or only with a certain subset. Information from a system subserving a particular function may undergo lateral inhibitory interactions within its patch system via the collateral network of common spiny neurons (Groves, 1983). These interactions would likely refine and sharpen patterns of input. It may be presumed that similar interactions occur in adjacent modules involving different afferent information. The lattice organization may rep- resent a structural scheme for interfacing different functional systems and thereby interrelating the consequences of processing these differentiated patterns of input.

\section{References}

Armstrong, D. M., V. M. Pickel, T. H. Joh, D. J. Reis, and R. J. Miller (1981) Immunocytochemical localization of catecholamine synthesizing cnzymes and neuropeptides in area postrema and medial nucleus tractus solitarius of rat brain. J. Comp. Neurol. 196: 505-517.

Beckstead, R. M. (1984) The thalamostriatal projection in the cat. J. Comp. Neurol. 223: 313-346.

Berman, A. L., and E. G. Jones (1982) The Thalamus and Basal Telencephalon of the Cat, University of Wisconsin Press, Madison.

Bolam, J. P., P. N. Izzo, and A. M. Graybiel (1985) Golgi-impregnation of the cat neostriatum combined with immunocytochemistry for substance P or met-enkephalin. Neurosci. Lett. (Suppl.) 22: S281.

Butcher, L. L., and G. K. Hodge (1976) Postnatal development of acetylcholinesterase in the caudate-putamen nucleus and substantia nigra of rats. Brain Res. 106: 223-240.

Chapin, J. K., M. Sadeq, W. K. Smith, D. S. Schlusselberg, and D. J. Woodward (1985) Three-dimensional reconstruction of the topography of cortico-striatal projections in the rat. Soc. Neurosci. Abstr. 11: 201.

DiFiglia, M., N. Aronin, and J. B. Martin (1982) Light and electron microscopic localization of immunoreactive leu-enkephalin in the monkey basal ganglia. J. Neurosci. 2: 303-320.

Donoghue, J. P., and M. Herkenham (1986) Neostriatal projections from individual cortical fields conform to histochemically distinct striatal compartments in the rat. Brain Res. 365: 397-403.

Gerfen, C. R. (1984) The neostriatal mosaic: Compartmentalization of cortico-striatal input and striato-nigral output. Nature $311: 461-$ 464.

Gerfen, C. R. (1985) The neostriatal mosaic. I: Compartmental organization of projections from the striatum to the substantia nigra in the rat. J. Comp. Neurol. 236: 454-476.

Goedert, M., P. W. Mantyh, S. P. Hunt, and P. C. Emson (1983) Mosaic distribution of neurotensin-like immunoreactivity in the cat striatum. Brain Res. 274: 176-179.

Goldman, P. S., and W. J. H. Nauta (1977) An intricately patterned pre-frontocaudate projection in the rhesus monkey. J. Comp. Neurol. 171: 369-386.

Goldman-Rakic, P. S. (1981) Prenatal formation of cortical input and development of cytoarchitectonic compartments in the neostriatum of the rhesus monkey. J. Neurosci. 1: 721-735.

Goldman-Rakic, P. S. (1982) Cytoarchitectonic heterogeneity of the primate neostriatum: Subdivision into island and matrix cellular compartments. J. Comp. Neurol. 205: 398-413.

Graybiel, A. M. (1983) Compartmental organization of the mammalian striatum. In Molecular and Cellular Interactions Underlying Higher Brain Functions: Progress in Brain Research, vol. 58, J. Changeux, J. Glowinski, M. Imbert, and F. E. Bloom, eds., pp. 247-256, Elsevier, New York.

Graybiel, A. M. (1984a) Correspondence between dopamine islands and striosomes of the mammalian striatum. Neuroscience 13:11571187.

Graybiel, A. M. (1984b) Neurochemically specified subsystems in the basal ganglia. In Functions of the Basal Ganglia (CIBA Foundation Symposium 107), D. Evered and M. O'Connor, eds., pp. 114-149, Pitman, London.

Graybiel, A. M., and M.-F. Chesselet (1984a) Compartmental distribution of striatal cell bodies expressing met-enkephalin-like immunoreactivity. Proc. Natl. Acad. Sci. USA 81: 7980-7984.

Graybiel, A. M., and M.-F. Chesselet (1984b) Distribution of cell bodies expressing substance $P$, enkephalin and dynorphin $B$ in kitten and cat striatum. Anat. Rec. 208: 64A.

Graybiel, A. M., and C. W. Ragsdale (1978) Histochemically distinct compartments in striatum of human, monkey and cat demonstrated by acetylthiocholinesterase staining. Proc. Natl. Acad. Sci. USA 75: 5723-5726.

Graybiel, A. M., C. W. Ragsdale, and S. Moon-Edley (1979) Compartments in the striatum of the cat observed by retrograde cell labeling. Exp. Brain Res. 34: 189-195.

Graybiel, A. M., C. W. Ragsdale, E. S. Yoneoka, and R. P. Elde (1981) An immunohistochemical study of enkephalins and other neuropep- 
tides in the striatum of the cat with evidence that the opiate peptides are arranged to form mosaic patterns in register with the striosomal compartments visible by acetylcholinesterase staining. Neuroscience 6: 377-397.

Graybiel, A. M., R. W. Baugham, and F. Eckenstein (1986) Cholinergic neuropil of the striatum observes striosomal boundaries. Nature 323: 625-627.

Groves, P. M. (1980) Synaptic endings and their postsynaptic targets in neostriatum: Synaptic specializations revcalcd from analysis of serial sections. Proc. Natl. Acad. Sci. USA 77: 6926-6929.

Groves, P. M. (1983) A theory of the functional organization of the neostriatum and the neostriatal control of voluntary movement. Brain Res. Rev. 5: 109-132.

Groves, P. M., and J. C. Linder (1983) Dendro-dendritic synapses in substantia nigra: Descriptions based on analysis of serial sections. Exp. Brain Res. 49: 209-217.

Groves, P. M., and C. J. Wilson (1980) Monoaminergic presynaptic axons and dendrites in locus coeruleus as seen in reconstructions of serial sections. J. Comp. Neurol. 193: 853-862.

Herkenham, M., and C. B. Pert (1981) Mosaic distribution of opiate receptors, parafascicular projections and acetylcholinesterase in rat striatum. Nature 291: 415-418.

Jimencz-Castcllanos, J., and A. M. Graybiel (1985) The dopaminecontaining innervation of striosomes: Nigral subsystems and their striatal correspondents. Soc. Neurosci. Abstr. 11: 1249.

Kalil, K. (1978) Patch-like termination of thalamic fibers in the putamen of the rhesus monkey: An autoradiographic study. Brain Res. 140: 333-339.

Kemp, J. M., and T. P. S. Powell (1970) The cortico-striate projection in the monkey. Brain 93: 525-546.

Kemp, J. M., and T. P. S. Powell (1971) The structure of the caudate nucleus of the cat: Light and electron microscopy. Phil. Trans. R. Soc. London [Biol.] 262: 383-401.

Malach, R., and A. M. Graybiel (1986) Mosaic architecture of the somatic sensory-recipient sector of the cat's striatum. J. Neurosci. 6: 3436-3458.

Marsden, C. D. (1982) The mysterious motor function of the basal ganglia. Neurology 32: 514-539.

Martone, M., P. M. Groves, S. J. Young, and D. M. Armstrong (1986) Three dimensional reconstruction of a striosomal network in the caudate nucleus of adult cats. Soc. Neurosci. Abstr. 12: 1543.

Moon-Edley, S., and M. Herkenham (1984) Heterogeneous dopaminergic projections to the neostriatum of the rat: Nuclei of origin dictate relationship to opiate receptor patches. Anat. Rec. 208: 120A.

Nastuk, M. A., and A. M. Graybiel (1985) Patterns of muscarinic cholinergic binding in the striatum and their relation to dopamine islands and striosomes. J. Comp. Neurol. 237: 176-194.

Nauta, W. J. H., and V. B. Domesick (1984) Afferent and efferent relationships of the basal ganglia. In Functions of the Basal Ganglia (CIBA Foundation Symposium 107), D. Evered and M. O'Connor, eds., pp. 3-23, Pitman, London.

O'Brien, T. F., D. A. Steindler, and N. G. F. Cooper (1986) A striatal mosaic revealed by lectin binding and tritiated fucose incorporation in the early postnatal mouse. Soc. Neurosci. Abstr. 12: 1543 .

Olson, L., A. Seiger, and K. Fuxe (1972) Heterogeneity of striatal and limbic dopamine innervation: Highly fluorescent islands in developing and adult rats. Brain Res. 44: 283-288.

Penney, G. R., C. J. Wilson, and S. T. Kitai (1984) The influence of neostriatal patch and matrix compartments on the dendritic geometry of spiny projection neurons in the rat as revealed by intracellular labeling with HRP combincd with immunocytochemistry. Soc. Neurosci. Abstr. 10: 514

Penney, G. R., S. Afsharpour, and S. T. Kitai (1986) The glutamate decarboxylase-, leucine-enkephalin-, methionine-enkephalin- and substance P-immunoreactive neurons in the neostriatum of the rat and cat: Evidence for partial overlap. Neuroscience 17: 1011-1045.

Pickel, V. M., K. K. Sumal, S. C. Beckley, R. J. Miller, and D. J. Reis (1980) Immunocytochemical localization of enkephalin in the neostriatum of rat brain: A light and electron microscopic study. J. Comp. Neurol. 189: 721-740.

Ragsdale, C. W., and A. M. Graybiel (1981) The frontostriatal projection in the cat and monkey and its relationship to inhomogeneities established by acetylcholinesterase histochemistry. Brain Res. 208: 259-266.

Ragsdale, C. W., and A. M. Graybiel (1984) Further observations on the striosomal organization of frontostriatal projections in cats and monkeys. Soc. Neurosci. Abstr. 10:514.

Sandell, J. H., A. M. Graybiel, and M.-F. Chesselet (1986) A new enzyme marker for striatal compartmentalization: NADPH diaphorase activity in the caudate nucleus and putamen of the cat. J. Comp. Neurol. 243: 326-334.

Selemon, L. D., and P. S. Goldman-Rakic (1985) Longitudinal topography and interdigitation of corticostriatal projections in the rhesus monkey. J. Neurosci. 5: 776-794.

Sternberger, L. A. (1979) Immunocytochemistry, Wiley, New York.

Takagi, H. (1986) Distribution of peptides in basal ganglia. Prog. Brain Res. 66: 35-72.

Tanaka, D., T. Gorska, and K. Dutkiewicz (1981) Corticostriate projections from the primary motor cortex in the dog. Brain Res. 209: 287-303.

Wilson, C. J., and P. M. Groves (1980) Fine structure and synaptic connections of the common spiny neuron of the ral neostriatum: A study employing intracellular injection of horseradish peroxidase. J. Comp. Neurol. 194: 599-615.

Wilson, C. J., P. M. Groves, S. T. Kitai, and J. C. Linder (1983) Threedimensional structure of dendritic spines in the rat neostriatum. J. Neurosci. 3: 383-398.

Yeterian, E. H., and G. W. Van Hoesen (1978) Cortico-striate projections in the rhesus monkey: The organization of certain corticocaudate connections. Brain Res. 139: 43-63.

Young, S. J., S. M. Royer, P. M. Groves, and J. C. Kinnamon (1987) Three-dimensional reconstructions from serial micrographs using the IBM PC. J. Electron Microsc. Tech. 6: 207-217. 